\title{
Proceso Estiloides Elongado: Criterios Diagnósticos y Diagnóstico Diferencial. A Propósito de un Caso
}

\author{
Elongated Styloid Process: Diagnostic Criteria and Differential Diagnosis. A Case Review
}

\author{
Rolando Morales E.* \& Mario Cantín L.*
}

MORALES, E. R. \& CANTíN, L. M. Proceso estiloides elongado: Criterios diagnósticos y diagnóstico diferencial. A propósito de un caso. Int. J. Odontostomat., 4(3):223-228, 2010.

RESUMEN: La elongación del proceso estiloides puede presentarse como una entidad patológica en el territorio craneocervical. Existe controversia en relación a los criterios para su diagnóstico, así como sus diagnósticos diferenciales. En el presente reporte se relata el caso de una paciente de 45 años con lumbagos crónicos, cefalea intermitente, dolor cervical y articular agudo, sin historia de quirúrgica o traumática craneocervical. Radiográficamente, destaca la elongación bilateral del proceso estiloides, determinando un diagnóstico presuntivo de Síndrome de Eagle. Se preconiza que los pacientes deben haber sido sometidos a tonsilectomía o trauma para padecer esta patología, condición ausente en este caso. La correcta discriminación de los signos y síntomas permite diferenciar la patología como síndrome estilohioideo. El presente reporte entrega los criterios a considerar en un paciente con el proceso estiloides elongado asociado a patología, con el fin de canalizar esa información hacia un diagnóstico certero.

PALABRAS CLAVE: proceso estiloides, diagnóstico diferencial, síndrome de Eagle, síndrome estilohioideo.

\section{INTRODUCCIÓN}

La elongación del proceso estiloides, condición asociada normalmente al síndrome de Eagle, corresponde a una entidad patológica descrita el año 1937 por el otorrinolaringólogo alemán Watt Eagle en dos pacientes tonsilectomizados que presentaron molestias a nivel cervical, faríngeo y oral, los cuales al examen radiológico evidenciaban una elongación del proceso estiloides del hueso temporal o bien la osificación del ligamento estilohioideo (Eagle, 1937). Sin embargo, esta variación anatómica del proceso estiloides ha sido observada desde mucho tiempo antes por diversos anatomistas y médicos a lo largo de la historia (Pierrakou \& Patsakas, 1987; Piagkou et al., 2009), la que es causada por alteraciones que afectan los componentes embrionarios derivados del segundo arco faríngeo, específicamente de su porción timpanohial y ceratohial (Cantín et al., 2007).

Este síndrome es considerado una entidad precisa en el diagnóstico diferencial de dolores de cabeza y cuello, constituyendo parte de los trastornos temporomandibulares (TTM) de origen musculoesquelético (Castillo et al., 2003).

El proceso estiloides es descrito como una proyección ósea cilíndrica que mide aproximadamente 2,5 a $3 \mathrm{~cm}$. Se encuentra en relación al hueso temporal en su porción timpánica, contiguo a las arterias carótidas externa e interna, a la salida del foramen estilomastoideo por donde emergen ramos del nervio facial y la arteria estilomastoidea. En esta proyección ósea tienen inserción tres músculos: el músculo estilohioideo, estilogloso y estilofaríngeo, incluyéndose además los ligamentos estilohioideo y el estilomandibular.

El síndrome de Eagle puede presentarse con o sin sintomatología. Del total de casos, un $4 \%$ presentan síntomas evidentes de su estado, encontrándose en un $98 \%$ de los pacientes una elongación bilateral de los procesos estiloides. Sin embargo, la sintomatología se presenta comúnmente de manera

\footnotetext{
* Escuela de Odontología, Facultad de Ciencias de la Salud, Universidad de Talca, Chile.

* Departamento de Ciencias Básicas y Biomédicas, Facultad de Ciencias de la Salud, Universidad de Talca, Chile.
} 
unilateral, siendo los síntomas bilaterales muy raros (Harma, 1967), y por lo general con el proceso estiloides derecho de mayor longitud.

La sintomatología característica de este síndrome, está determinada por un dolor faríngeo persistente el cual puede ser irradiado hacia la región del oído (Dolan et al., 1984; Sandev \& Sokler, 2000), existiendo además una variante vascular denominada como síndrome de la arteria carótida o carotidinia (Eagle, 1948), la cual no guarda relación con una tonsilectomía, sino que a la irritación y compresión persistente de la arteria carótida interna por el proceso estiloides elongado al girar la cabeza, pudiendo resultar incluso en un accidente isquémico transitorio o un accidente vascular encefálico (Farhat et al., 2009).

Epidemiológicamente, la incidencia del síndrome de Eagle es muy controversial (Rogers \& Chang, 2007). Se ha descrito que entre un $4 \%$ (Eagle, 1937) y $28 \%$ (Kaufman et al., 1970) de la población puede presentar el proceso estiloides elongado, siendo más prevalente en mujeres (Correl \& Wescott, 1982), pero con mayor longitud en los hombres (Fuentes et al., 2007), iniciando su elongación entre la tercera y cuarta décadas de vida. Esto posiblemente debido a mostrar una forma romanizada y una mayor deposición de sales de calcio en los ligamentos y los procesos (Unlu et al., 2008). No existen datos de prevalencia de proceso estiloides elongados en niños (Quereshy et al., 2001).

Eagle (1937) en su investigación describió dos síndromes por separado, caracterizando el síndrome clásico (la presentación más común) como el que ocurre siempre después de una tonsilectomía (Leong et al., 2007) y está definido sintomatológicamente por la sensación de cuerpo extraño en la faringe, disfagia, odinofagia y otalgia (Paraskevas et al., 2009), caracterizada por un dolor intenso y crónico (Hossein et al., 2010). El segundo es el síndrome de la arteria carótida, que se presenta con o sin tonsilectomia. En este caso el proceso estiloides elongado comprime la arteria carótida interna o externa, localizando el dolor en la región parietal o alrededor del ojo, pudiendo presentar mareos, pérdida transitoria de la visión y síncope.

Según Camarda et al. (1989), la clasificación para el dolor cervicofaríngeo asociado a la elongación del proceso estiloides se divide en 3 distintas clases: la primera que es el síndrome de Eagle propiamente tal, el que requiere la presencia de una cirugía a nivel cervical como una tonsilectomía o trauma, junto a la presencia de do- lor al realizar la palpación clínica de la elongación del proceso estiloides intraoralmente, más un examen imagenológico que demuestre la elongación del proceso, lo que provee un diagnóstico certero de este (Weidenbecher et al., 2006; Piagkou et al.). La segunda entidad es el síndrome estilohioideo, en donde el paciente relata sintomatología, no existe un trauma o cirugía previa, sin embargo en el examen radiográfico existe presencia de un proceso estiloides elongado o su osificación, dentro de una edad joven o adulta (alrededor de los 40 años de edad). En el tercer caso existe otra entidad que es el síndrome pseudoestilohioideo, donde el paciente describe los síntomas clásicos del síndrome de Eagle pero no existe una presencia evidente, clínica ni radiográfica de alguna elongación u osificación, siendo este consecuencia del envejecimiento por pérdida de elasticidad de los tejidos, con frecuencia manifestada por tendinitis y reacciones inflamatorias a nivel del territorio inervado por el nervio glosofaríngeo, lo cual causa dolor (Bafaqeeh, 2000).

A continuación, reportamos el caso de un paciente que presenta ambos procesos estiloides elongados, acompañado de sintomatología, en donde una correcta anamnesis y, análisis de signos y síntomas, junto a la correcta utilización de los criterios diagnósticos, permiten un diagnóstico diferencial adecuado.

\section{CASO CLÍNICO}

Paciente, género femenino, 45 años de edad acude a la consulta dental por rehabilitación protésica a nivel oral. Presenta antecedentes médicos de hipertensión, dislipidemia, lumbagos crónicos y depresión, la cual está en tratamiento. Relata no haber recibido ningún tipo de cirugía a nivel corporal o a nivel cervicofacial. La paciente manifiesta padecer de dolores agudos a nivel cervical al momento de realizar movimientos de lateralidad con la cabeza y episodios dolorosos a nivel cervical por las noches, además de una cefalea intermitente los últimos meses.

Se realiza examen extraoral con palpación a musculatura oral y perioral presentando dolor a nivel de músculos temporal anterior, pterigoideo lateral, esternocleidomastoideo bilateralmente, y a nivel de los músculos cervicales posteriores, calificado como nivel 2 por la paciente en una escala de 0 a 3 , donde 0 no existe dolor, y 3 es un dolor intenso. Al examen articular presenta dolor en ATM derecha en apertura, con desviación en apertura y cierre. 
Al examen intraoral presenta pérdida múltiple de piezas dentarias superiores e inferiores, con antecedentes de extracciones traumáticas. Además la paciente relata no haber portado prótesis dental previamente.

Se le solicita radiografía panorámica de rutina, donde destaca como hallazgo radiográfico la elongación bilateral de los procesos estiloides de la paciente, midiendo $35 \mathrm{~mm}$ el proceso izquierdo y 40 $\mathrm{mm}$. el derecho (Fig. 1). Se remite a la paciente a interconsulta a especialista en cirugía maxilofacial, donde se solicitan nuevos exámenes radiográficos: radiografía antero posterior y de perfil, donde se hace evidente nuevamente la elongación de los procesos estiloides de forma bilateral (Figs. 2 y 3 ).

Se le informa a la paciente de su condición, las posibilidades de tratamiento quirúrgico o farmacológico. Debido a los dolores crónicos que presentaba, se deriva a la paciente a tratamiento kinesiológico, siendo confirmado su diagnóstico cervical y dándosele tratamiento para el dolor con antiinflamatorios y analgésicos. La paciente luego de 3 meses de tratamiento ha manifestado una disminución en su dolor y molestias a nivel cervical.

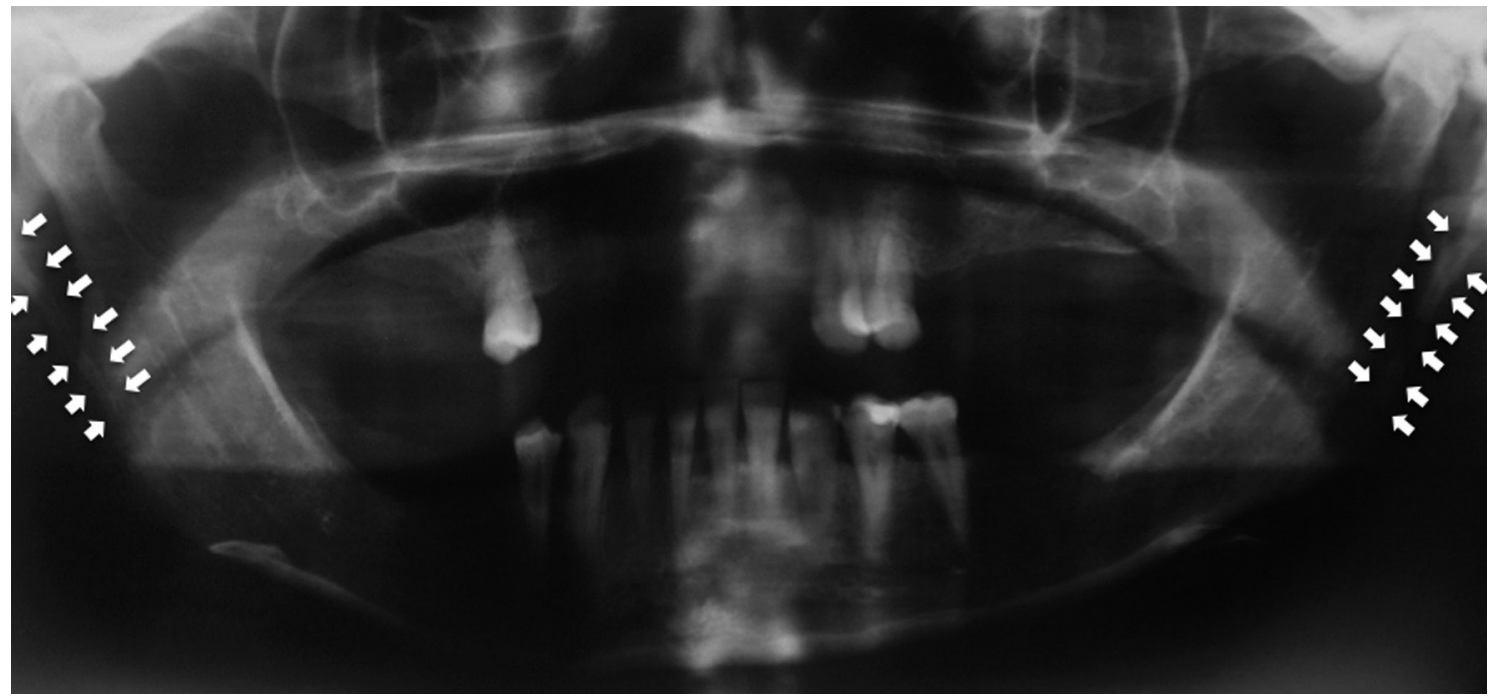

Fig. 1. Ortopantomografía donde se muestran los procesos estiloides elongados bilateralmente.

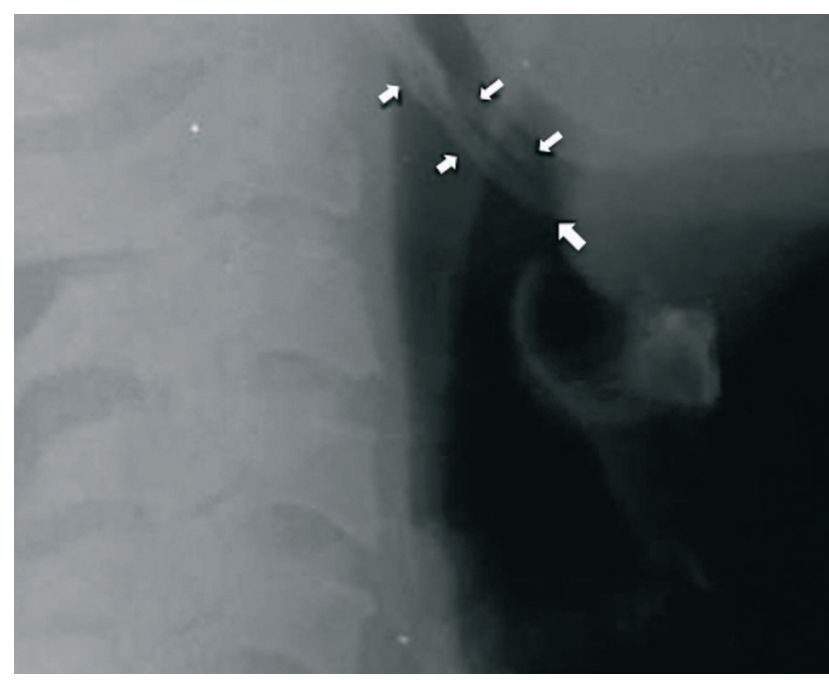

Fig. 2. Radiografía lateral donde se aprecia el elongamiento de los procesos estiloides bilateralmente y su grado de angulación.

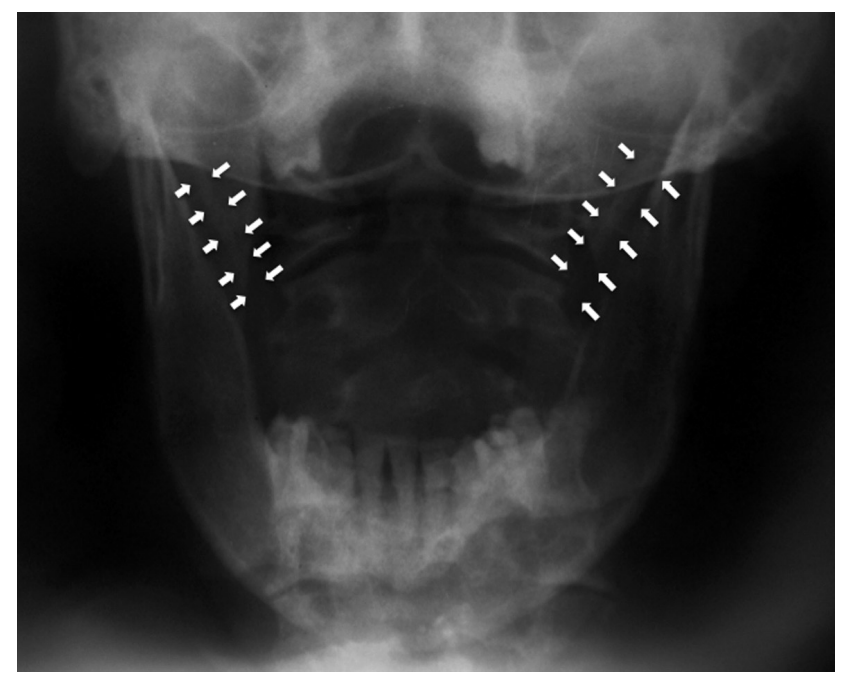

Fig. 3. Radiografía antero-posterior de cráneo a boca abierta que muestra el proceso estiloides elongado bilateralmente. 


\section{DISCUSIÓN}

El síndrome de Eagle se caracteriza por ser una entidad patológica escasamente definida en cuanto a su sintomatología, por lo que es un tanto difuso el realizar el diagnóstico preciso de esta patología, a menos que se tenga un claro indicio de que existe una alteración en las estructuras músculo esqueléticas del paciente.

A menudo se presenta con dolor difuso en la faringe, dolor facial, otalgia y dificultad para tragar. Los síntomas clínicos son causados por la compresión de los nervios adyacentes, principalmente el nervio glosofaríngeo, el ramo inferior del nervio trigémino y el nervio cuerda del tímpano. Las otras causas posibles del dolor pueden ser debido a la proliferación de tejido de granulación después de una fractura traumática del proceso estiloides (Balasubramanian, 1964) o incidencias sobre la arteria carótida (Moffat et al., 1977).

Esta sintomatología puede ser confundida con otras patologías de tipo articular en relación a la ATM, ya que la paciente presenta una gran pérdida de piezas dentarias lo que hace pensar en problemas de tipo mecánico o de compresión sobre los tejidos de la ATM con los consiguientes problemas a nivel muscular, ya que al existir una inestabilidad mandibular debido a la pérdida de la mesa oclusal posterior y dimensión vertical, esta debe ser compensada por la acción muscular de los músculos craneomandibulares adyacentes a la ATM y a la mandíbula provocando inflamación y dolor, todo lo anterior considerando además que la paciente no ha sido portadora de prótesis dental previa.

Además, se deben tener en cuenta otros diagnósticos diferenciales tales como dolor de cabeza (los que incluyen migraña, cefaleas en racimo, cefaleas por tensión crónica, cervicocefalalgias, carotidinias, dolor facial atípico y hemicránea paroxística) (Casale et al., 2008); dolor facial (incluyendo disfunción de la articulación temporomandibular, síndrome de dolor por disfunción miofascial, neuralgia del nervio trigémino, glosofaríngeo, laríngeo superior y occipital, del ganglio pterigopalatino y geniculado, clic mandibular, molares no erupcionados o impactados, prótesis dentales defectuosas, enfermedad de las glándulas salivales y dolor dentario) (Nishihara et al., 1986; Chase et al., 1986; Aral et al., 1997; Piagkou et al.); dolor cervical (enfermedad degenerativa discal, reflujo laringofaríngeo crónico) (Casale et al.; GelabertGonzález \& García-Allut, 2008); y patologías que afecten al oído, nariz y garganta (tonsilitis crónica, cálculos tonsilares, espasmo del músculo constrictor faríngeo, otitis, mastoiditis, fractura del hueso hioides, bursitis del hamulus pterigoideo, entre otras) (Piagkou et al.).

Es importante de considerar en el diagnóstico de la paciente la presencia de lumbago crónico, lo cual es un indicio además de problemas a nivel de la columna vertebral, los cuales pueden generar alteraciones a nivel de la columna cervical, afectando la musculatura craneocervical.

Lo que más destaca del caso, es que la paciente no ha sido sometida a tonsilectomia ni a cirugía alguna a nivel cervical, o ha padecido traumatismos previos, por lo que esto determinaría su diagnostico como síndrome estilohioideo (según la clasificación de Camarda et al.), haciendo el diferencial con el síndrome de Eagle, que está caracterizado por que los pacientes han sido tonsilectomizados (Leong et al., 2007), situación clave para la manifestación de los signos y síntomas característicos de esta presentación clásica. Ya que la paciente relata la sintomatología, y en el examen radiográfico existe presencia de un proceso estiloides elongado o su osificación, y presenta una edad adulta (alrededor de los 40 años de edad). Por otra parte, la paciente no presenta trastornos degenerativos a nivel muscular o articular, ni tampoco una edad avanzada para definir un diagnóstico de síndrome pseudoestilohioideo.

Pese a que no se realizó el examen de palpación del proceso estiloides elongado a través de la fosa tonsilar, se realizó un examen físico segmentario detallado de cabeza y cuello, tanto por el personal odontológico como por la kinesióloga. El examen de la fosa tonsilar es sugerido como una alternativa táctil de examen (Balbuena et al., 1997), donde se coloca el dedo índice en el pilar anterior de la fosa, previa anestesia local ( $1 \mathrm{ml}$ de lidocaina al $2 \%$ ), desde la base hasta la parte superior incluyendo la superficie de la tonsila y luego la fosa tonsilar, siempre y cuando no exista presencia de tonsilas (Prasad et al., 2002). El proceso estiloides se palpa como una cuerda ósea o una punta, acompañado por el dolor del paciente. También se puede palpar una masa en el ángulo de la mandíbula y esto genera dolor. Hampf et al. (1986) determinaron que el proceso estiloides sólo se puede palpar en la fosa tonsilar si es más larga de $7,5 \mathrm{~cm}$. Sin embargo, un proceso estiloides mucho más corto también puede producir síntomas (Ghosh \& Dubey, 1999).

Cabe destacar que en el caso clínico el hecho de observar en el estudio radiográfico la presencia de procesos estiloides elongados fue un hallazgo radiográfico, ya que la radiografía panorámica constituye una parte 
del arsenal de instrumentos diagnósticos solicitados para el diagnóstico integral de la paciente. Esto presupone la importancia de los exámenes imagenológicos, tales como radiografía lateral del cuello, ortopantomografía o una tomografía computarizada (TC) (Prasad et al.; Piagkou et al.). En la ortopantomografía, el proceso estiloides es típicamente considerado como elongado si su longitud es superior a $1 / 3$ de la longitud de la rama de la mandíbula (Prasad et al.). Una ventaja de la ortopantomografía es que toda la longitud del proceso estiloides es visible y su desviación se puede medir con bastante precisión (Ghosh \& Dubey). Estos exámenes imagenológicos van a permitir al clínico el considerar una etiología musculoesquelética de los dolores cervicales y la presencia o no de alteraciones en la forma de los tejidos normales de la cabeza y del cuello, ya que previo a la toma radiográfica muchas de las dolencias musculares tenían una explicación a nivel de sobrecarga de músculos masticadores o bien a problemas asociados al stress que está sometida la paciente por su depresión, y no a una causa anatómica. Además, la ortopantomografía es un método simple, rápido y accesible a cualquier profesional que esté en la pesquisa de esta patología.
En conclusión, es importante señalar que el proceso estiloides elongado no significa necesariamente síndrome de Eagle o sus variantes, ya que la mayoría de las personas que presenta estavariación anatómica no presentan síntomas. En los casos que su presencia genera sintomatología, los posibles diagnósticos asociados a un proceso estiloides elongado o bien una calcificación del ligamento estilohioideo deben estar basados en una historia clínica fiel, un examen clínico cuidadoso, tanto físico como imagenológico, en donde se incluya el máximo de precisión en el registro e interpretación de los datos, además de la realización de las interconsultas a profesionales especializados que puedan cooperar en la solución del caso para así lograr un correcto diagnóstico y tratamiento de la patología que aqueja al paciente que busca una respuesta a su dolencia.

AGRADECIMIENTOS. A aquel que tiene un Nombre por sobre todo nombre, que me permitió mirar más allá de lo evidente; al Dr. Mario Cantín que guió mis ansias de investigar, al Dr. Andrés Schlosser y al Dr. Nicolás Ríos por su valiosa cooperación en el ámbito clínico.

MORALES, E. R. \& CANTíN, L. M. Elongated styloid process: diagnostic criteria and differential diagnosis. A case review. Int. J. Odontostomat., 4(3):223-228, 2010.

ABSTRACT: The elongation of the styloid process may occur as a pathologic entity in the craniocervical area. There is controversy regarding the criteria for its diagnosis and its differential diagnosis. In this report we present the case of a 45 year old female with chronic low back pain, intermittent headache, neck pain and acute articular, no history of surgical or traumatic in the craniocervical area. Radiographically, bilateral elongation of the styloid process were detected, determining a presumptive diagnosis of Eagle syndrome. It requires that patients must have undergone tonsillectomy or trauma to suffer this disease, a condition absent in this case. The correct discrimination of the signs and symptoms distinguish this condition as stylohyoid syndrome. This report delivers the criteria to consider in a patient with elongated styloid process associated with pathology, to use that information and give an accurate diagnosis.

KEY WORDS: styloid process, differential diagnosis, Eagle syndrome, stylohyoid syndrome.

\section{REFERENCIAS BIBLIOGRÁFICAS}

Aral, I. L.; Karaca, I. \& Güngör, N. Eagle's syndrome masquerading as pain of dental origin. Case report. Aust. Dent. J., 42(1):18-9, 1997.

Bafaqeeh, S. A. Eagle syndrome: Classic and carotid artery types. J. Otolaryngol., 29:88-94, 2000.

Balasubramanian, S. The ossification of syloid ligament and its relation to facial pain. Br. Dent. J.,116:108-11, 1964.

Balbuena, L. Jr.; Hayes, D.; Ramirez, S. G. \& Johnson, R. Eagle's syndrome (elongated styloid process) South Med. J., 90(3):331-4, 1997.
Camarda, A. J.; Deschamps, C. \& Forest, D. I. Stylohyoid chain ossification: A discussion of etiology. Oral Surg. Oral Med. Oral Pathol., 67:508-14, 1989.

Cantín, L. M.; Suazo, G. I., Venegas, R. B.; Zavando, M. D. \& Muñoz, C. R. Síndrome de Eagle bilateral, reporte de un caso. Int. J. Odontostomat., 1:141-5, 2007.

Casale, M.; Rinaldi, V.; Quattrocchi, C.; Bressi, F.; Vincenzi, B.; Santini, D.; Tonini, G. \& Salvinelli, F. Atypical chronic head and neck pain: Don't forget Eagle's syndrome. Eur. Rev. Med. Pharmacol. Sci, 12:131-3, 2008. 
Castillo, C. E.; Mantilla, J. C.; Sandoval, G .P. \& Ramírez L. M. Síndrome de Eagle: tomografía del proceso estiloideo alongado. Acta de otorrinolaringología \& cirugía de cabeza y cuello, 31(3):89-96, 2003.

Chase, D. C.; Zarmen, A.; Bigelow, W. C. \& McCoy, J. M. Eagle's syndrome: a comparison of intraoral versus extraoral surgical approaches. Oral Surg. Oral Med. Oral Pathol., 62(6):625-9, 1986.

Correll, R. W. \& Wescott, W. Eagle's syndrome diagnosed after history of headache, dysphagia, otalgia, and limited neck movement. J. Am. Dent. Assoc., 104:4912, 1982.

Dolan, E. A.; Mullen, J. B. \& Papayoanou, J. Styloidstylohyoid syndrome in the differential diagnosis of atypical facial pain. Surg. Neurol., 21:291-4, 1984.

Eagle, W. W. Elongated styloid processes: report of two cases. Arch. Otolaryngol., 25:584-7, 1937.

Eagle, W. W. Elongated styloid process, further observations and a new syndrome. Arch. Otolaryngol., 47:630-40, 1948.

Farhat, H. I.; Elhammady, M. S.; Ziayee, H.; Aziz-Sultan, M. A. \& Heros, R. C. Eagle syndrome as a cause of transient ischemic attacks. J. Neurosurg., 110:90-3, 2009.

Fuentes, F. R.; Oporto, V. G.; Garay, C. I.; Bustos, M. L.; Silva, M. H. \& Flores, F. H. Proceso estiloides en una muestra de radiografías panorámicas de la ciudad de Temuco-Chile. Int. J. Morphol., 25(4):729-33, 2007.

Gelabert-Gonzalez, M. \& Garcia-Allut, A. Eagle syndrome. An unusual cause of neck pain. Neurocirugia (Astur), 19:254-6, 2008.

Ghosh, L. M. \& Dubey, S. P. The syndrome of elongated styloid process. Auris Nasus Larynx, 26:169-75, 1999.

Hampf, G.; Aalberg, V.; Tasanen, A. \& Nyman, C. A holistic approach to stylalgia. Int. J. Oral Maxillofac. Surg., 15:549-52, 1986.

Harma, R. Stylalgia: Clinical experience of 52 cases. Acta Otolaryngol., 224:149-55, 1967.

Hossein, R.; Kambiz, M.; Mohammad, D. \& Mina, N. Complete recovery after an intraoral approach for Eagle syndrome. J. Craniofac. Surg., 21(1):275-6, 2010.

Kaufman, S. M.; Elzay, R. P. \& Irish, E. F. Styloid process variation. Radiologic and clinical study. Arch. Otolaryngol., 91:460-3, 1970.
Leong, S. C.; Karkos, P. D.; Papouliakos, S. M. \& Apostolidou, M. T. Unusual complications of tonsillectomy: a systematic review. Am. J. Otolaryngol., 28(6):419-22, 2007.

Moffat, D. A.; Ramsden, R. T. \& Shaw, H. T. The styloid process syndrome. J. Laryngol. Otol., 91:279-94, 1977.

Nishihara, K.; Hanakita, J.; Kinuta, Y.; Kondo, A.; Yamamoto, Y. \& Kishimoto, S. Three cases of Eagle's syndrome. No Shinkei Geka, 14:441-5, 1986.

Paraskevas, G. K.; Raikos, A.; Lazos, L. M. \& Kitsoulis, P. Unilateral elongated styloid process: a case report. Cases J., 2:9135, 2009.

Piagkou, M.; Anagnostopoulou, S.; Kouladouros, K. \& Piagkos, G. Eagle's syndrome: a review of the literature. Clin. Anat., 22(5):545-58, 2009.

Pierrakou, E. D. \& Patsakas, A. J. Eagle's syndrome. Review of the literature. Hell. Stomatol. Chron., 31(2):111-6, 1987.

Prasad, K. C.; Kamath, M. P.; Reddy, K. J.; Raju, K. \& Agarwal, S. Elongated styloid process (Eagle's syndrome): a clinical study. J. Oral Maxillofac. Surg., 60(2):171-5, 2002.

Quereshy, F. A.; Gold, E. S.; Arnold, J. \& Powers, M. P. Eagle's syndrome in an 11-year-old patient. J. Oral Maxillofac. Surg., 59:94-7, 2001.

Rogers, K. \& Chang, H. Eagle's syndrome: A case report. Columbia Dent. Rev., 11:12-4, 2007.

Sandev, S. \& Sokler, K. Styloid process syndrome. Acta Stomatol. Croat., 34:451-6, 2000.

Unlu, Z.; Orguc, S.; Eskiizmir, G.; Aslan, A. \& Bayindir, P. Elongated styloid process and cervical spondylosis. Clinical Medicine: Case Reports, 1:57-64, 2008.

Weidenbecher, M.; Schick, B. \& Iro, H. Styloid syndrome and its treatment. Laryngorhinootologie, 85:184-90, 2006.

Dirección para correspondencia:

Rolando Morales E.

Escuela de Odontología

Facultad de Ciencias de la Salud

Universidad de Talca

Avenida Lircay sin número

Talca - CHILE Recibido :13-09-2010

Email: rolamoraes@gmail.com 